\title{
Socio-demographic and modifiable risk factors of diabetes and hypertension among resource constrained patients from rural areas in Mdantsane Township in South Africa
}

\section{Lettilia Xhakaza ${ }^{1}$, Zainonesa Abrahams-October ${ }^{1}$, Mohammedmekin Mohammedseid Mohammednur ${ }^{1}$, Brendon Pearce ${ }^{1}$, Oladele Vincent Adeniyi ${ }^{2}$, Rabia Johnson ${ }^{3,4}$, Mongi Benjeddou ${ }^{1}$}

1. Precision Medicine Unit, Department of Biotechnology, Faculty of Natural Sciences, University of the Western Cape, Robert Sobukwe Road, Bellville, 7535, South Africa.

2. Department of Family Medicine, Walter Sisulu University, East London, South Africa.

3. South African Medical Research Council, Parow, Cape Town, South Africa.

4. Division of Medical Physiology, Faculty of Medicine and Health Sciences, Stellenbosch University, Tygerberg 7505, South Africa. rabia.johnson@mrc.ac.za

\begin{abstract}
Background: Recently, developing countries have shown a dramatic increase in non-communicable diseases (NCDs). The burden of NCDs in South Africa has increased over the past years resulting in an estimated 37\% of all- cause mortality and $16 \%$ of disability-adjusted life years. Currently, diabetes mellitus (DM) and hypertension (HTN) are the two most prevalent NCDs associated with the rapid increase in mortality.

Objective: To demonstrate the socio-demographic and modifiable risk factors of diabetes mellitus (DM) and hypertension (HTN) among South African adults.

Methods: A cross-sectional analytical study was conducted in the Cecilia Makiwane Hospital serving the residents of Mdantsane. Relevant socio-demographic data, anthropometric measurements, triplicate blood pressure, fasting blood glucose and lipogram analysis were obtained from 265 outpatients.

Results: Multivariate anlysis shows that; salt intake, smoking, elevated triglycerides and decreased high-density lipoprotein levels were significantly associated with DM with adjusted odds ratio of $0.18(\mathrm{p}=0.002), 0.26(\mathrm{p}=0.048), 2.19(\mathrm{p}=0.006)$ and $0.38(\mathrm{p}=0.001)$, respectively. Overweight and obesity were significantly associated with hypertension with odds ratio of 0.03 $(\mathrm{p}=0.01)$ and $0.06(\mathrm{p}=0.006)$, respectively.

Conclusion: The burden of DM and HTN on society can be drastically reduced with simple lifestyle changes, development of preventative strategies, large-scale screening and better disease management in South Africa.

Keywords: Diabetes, hypertension, rural areas, Mdantsane Township, South Africa.

DOI: https://dx.doi.org/10.4314/ahs.v20i3.41

Cite as: Xhakaza L, Abrahams-October Z, Mohammednur MM, Pearce B, Adeniyi OV, Johnson R, et al. Socio-demographic and modifiable risk factors of diabetes and hypertension among resource constrained patients from rural areas in Mdantsane Township in South Africa. Afri Health Sci. 2020;20(3): 1344-1354. https:/ / dx.doi.org/10.4314/ahs.v20i3.41
\end{abstract}

\section{Introduction}

Non-Communicable Diseases (NCDs) have currently been identified as the leading cause of death worldwide. In the past decade developing countries have shown a dramatic increase in NCDs ${ }^{1,2}$. The burden

\section{Corresponding author:}

Mongi Benjeddou,

Precision Medicine Unit, Department of

Biotechnology, Faculty of Natural Sciences, University of the Western Cape, Robert Sobukwe

Road, Bellville, 7535, South Africa.

Tel: (+27)219592080

Email: mbenjeddou@uwc.ac.za of NCDs in South Afica has increased over the past years resulted in an estimated $37 \%$ of all cause mortality and $16 \%$ of disability-adjusted life years ${ }^{3-5}$. Currently, Diabetes Mellitus (DM) and hypertension (HTN) are the two most prevalent NCDs associated with the rapid increase in mortality ${ }^{6-10}$.

DM is defined as a chronic health condition associated with elevated blood sugar levels ${ }^{11 ; 12}$, whilst HTN is characterized by a systolic blood pressure $\geq 140 \mathrm{mmHg}$ and a diastolic blood pressure $\geq 90 \mathrm{mmHg}^{2}$. DM often co-exists with HTN since they both share common disease mechanisms and, in some instances, the one condition exacerbates the other ${ }^{13}$. Currently, 425 million people are diagnosed with diabetes, whilst it is estimated that over a billion people worldwide are af-

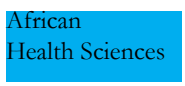

(C) 2020 Xhakaza L et al. Licensee African Health Sciences. This is an Open Access article distributed under the terms of the Creative commons Attribution License (https://creativecommons.org/licenses/BY/4.0), which permits unrestricted use, distribution, and reproduction in any medium, provided the original work is properly cited. 
fected with HTN ${ }^{2,14}$. Both diseases have strongly been associated with an increased risk of kidney failure, obesity, stroke, blindness, nerve damage and cardiovascular disease (CVD) ${ }^{2,15-18}$.

DM and HTN have been shown to have a major impact on public health funding consuming a significant proportion of public health spending ${ }^{7}$. However, these are described as lifestyle diseases, thus they can be prevented or managed by drugs and lifestyle modification ${ }^{19-21}$. Modifiable risk factors associated with DM and HTN include: tobacco use, alcohol consumption, physical activity and unhealthy diets ${ }^{22}$. An unhealthy diet is defined, according to Forouzanfar et al ${ }^{22}$, as a diet which is high in sodium, low in vegetables, low in fruit, low in whole grains, low in nuts and seeds, and low in seafood omega-3. Low- and middle-income countries are the most affected by these risk factors ${ }^{23,24}$.

The African region has been identified to have the highest burden of DM and HTN ${ }^{30 ; 14}$. In Africa, DM is estimated at 15.5 million adults aged between $20-79$ years $^{31}$ and HTN is estimated at $46 \%$ in adults $>25$ years 30 . The ever-increasing numbers of individuals diagnosed with these diseases are of great concern across the world especially in middle- and low-income countries ${ }^{30}$. The present study highlights the burden and associated risk factors of DM and HTN in Mdantsane, a resource constrained township of South Africa.

In South Africa, the burden of NCDs is predicted to increase substantially in the next decades if the necessary preventative measures are not taken ${ }^{25}$. Furthermore, strategies need to be implemented to effectively manage these diseases ${ }^{26}$. Currently, there are limited studies exploring the prevalence and risk factors of DM and HTN in South Africa, especially within the economically disadvantaged population. The aim of this study was to investigate the socio-demographic and modifiable risk factors of diabetes and hypertension in one of the rural areas in South Africa, with resource constrained patients. Modifiable risk factors found significantly associated with diabetes and/or hypertension could be used to promote health education as a primary intervention.

\section{Methods}

\section{Study area and design}

A cross-sectional descriptive study was conducted in the Cecilia Makiwane Hospital (Mdantsane, South Af- rica) from July 2017 - October 2017. Mdantsane is located in the Buffalo Municipality and is a low-income residential township with a population of approximately $150000^{27}$. The objectives of the study were explained to all participants and each participant signed a consent form indicating voluntary participation in the study. Information sheets were provided in both English and IsiXhosa languages. Prior to sampling, participants underwent physical examination and medical history was recorded.

\section{Study population and sampling}

Inclusion criteria for participants in this study were individuals aged $\geq 18$ years and have been diagnosed with hypertension and/or diabetes for more than a year prior to the study. Exclusion criteria included pregnant women, patients diagnosed with type 1 diabetes and acute illnesses. Age, sex, monthly income, level of education, lifestyle profile (i.e. physical activity, smoking status, alcohol and diet), and family history of disease prevalence were obtained through interview from all of the participants. The use of anti-hypertensive and antidiabetic medications along with the durations of disease(s) was obtained from the patients' medical records. Eligible participants $(\mathrm{N}=265)$ were recruited sequentially at the study setting over the study period.

\section{Data collection}

A trained research nurse conducted anthropometric measurements of: weight to the nearest $0.1 \mathrm{~kg}$, height to the nearest of $0.1 \mathrm{~cm}$ using a stadiometer, waist circumference, hip circumference and upper-arm circumference was measured using a tape measure. Measurements were taken with all participants wearing minimal clothing and no shoes. Blood pressure (BP) was measured using a validated automated digital blood pressure monitor (Microlife ${ }^{\circledR}$ BP A100 Plus). BP was recorded in triplicate and the average was used for analysis. Blood glucose was measured using Accutrend ${ }^{\circledR}$ test strips. Body Mass Index (BMI) for each patient was calculated as weight $(\mathrm{kg})$ divided by height $(\mathrm{m} 2)$ and was categorised based on WHO criteria: underweight $\left(<18.5 \mathrm{~kg} / \mathrm{m}^{2}\right)$, normal weight $\left(18.5-24.9 \mathrm{~kg} / \mathrm{m}^{2}\right)$, overweight (25.0-29.9 kg/ m${ }^{2}$ ) and obese (30 or greater $\mathrm{kg} /$ $\mathrm{m}^{2}$ ). Patients with systolic BP (SBP) of $\geq 140 \mathrm{mmHg}$ and $\geq 90$ diastolic BP (DBP) were identified as hypertensive and patients with systolic and diastolic BP below $140 \mathrm{mmHg}$ and $90 \mathrm{mmHg}$ respectively were identified as normotensive. 


\section{Laboratory assessment}

Fasting venous blood was obtained for all patients. The lipid profile which includes: total cholesterol (TC), triglycerides (TG), low-density lipoprotein (LDL) and high-density lipoprotein (HDL) for each participant was categorized according to the guidelines of the Heart and Stroke Foundation of South Africa ${ }^{5}$, ${ }^{28}$. In addition to this, the glycosylated haemoglobin (HbA1c) was assayed from blood samples of diabetic participants ${ }^{29}$. All blood samples were sent to the clinical laboratory centre, i.e. National Health Laboratory Services (NHLS) of Cecilia Makiwane hospital and the East London private hospital.

\section{Statistical analysis}

Statistical analysis was performed using Statistical Package for Social Science (SPSS) version 25 for Windows
(SPSS Inc., Chicago, IL, USA). The clinical laboratory data and anthropometric measurements were expressed as mean (n) \pm standard deviation (SD). Differences between groups were assessed using chi-square test for statistical significance. Risk factors associated with DM and HTN are presented as percentages with the odds ratios (ORs) and 95\% confidence intervals (CIs). The p-value $\leq 0.05$ were considered statistically significant.

\section{Results}

In the study cohort, a total of 265 outpatients (of which $n=175$ were female and $n=90$ were male) were interviewed during a 3-month study (Table 1). The mean ages of men and women were $59.96 \pm 11.19$ and 61.32 \pm 11.60 years, respectively. Other demographic, anthropometric and clinical laboratory measurements of the study participants are indicated in (Table 1).

Table 1: Characteristics of the study subjects in Mdantsane, Eastern Cape (July 2018).

\begin{tabular}{|c|c|c|c|}
\hline Parameter & $\begin{array}{l}\text { Female } \\
(n=175)\end{array}$ & Male $(n=90)$ & Total $(n=265)$ \\
\hline Age (years) & $59.96 \pm 11.19$ & $61.32 \pm 11.6$ & $60.42 \pm 11.32$ \\
\hline Weight (Kg) & $87.45 \pm 21.46$ & $81.62 \pm 16.06$ & $85.46 \pm 19.94$ \\
\hline Height (cm) & $159.93 \pm 6.22$ & $168.10 \pm 11.66$ & $162.72 \pm 9.30$ \\
\hline BMI $\left(\mathrm{Kg} / \mathrm{m}^{2}\right)$ & $34.18 \pm 8.27$ & $29.77 \pm 12.93$ & $32.68 \pm 10.29$ \\
\hline $\operatorname{HbA1c}(\%) *$ & $\begin{array}{c}10.40 \pm 2.80 \\
(\mathrm{n}=85)\end{array}$ & $\begin{array}{c}10.48 \pm 3.91 \\
(\mathrm{n}=32)\end{array}$ & $\begin{array}{c}10.42 \pm 3.12 \\
(\mathrm{n}=117)\end{array}$ \\
\hline FBG $(\mathrm{mmol} / \mathrm{l}) *$ & $\begin{array}{c}12.65 \pm 5.11 \\
(\mathrm{n}=85)\end{array}$ & $\begin{array}{c}13.11 \pm 3.92 \\
(\mathrm{n}=32)\end{array}$ & $\begin{array}{c}12.78 \pm 4.80 \\
(\mathrm{n}=117)\end{array}$ \\
\hline $\begin{array}{l}\text { Systolic blood } \\
\text { pressure (mmHg) }\end{array}$ & $155.67 \pm 20.53$ & $157.04 \pm 21.65$ & $156.14 \pm 20.88$ \\
\hline $\begin{array}{l}\text { Diastolic blood } \\
\text { pressure (mmHg) }\end{array}$ & $92.56 \pm 13.18$ & $93.87 \pm 13.45$ & $93.00 \pm 13.26$ \\
\hline Heart rate (pbm) & $84.14 \pm 13.60$ & $80.01 \pm 14.43$ & $82.74 \pm 13.99$ \\
\hline TC (mmol/L) & $5.02 \pm 1.27$ & $4.53 \pm 1.14$ & $4.86 \pm 1.25$ \\
\hline HDL (mmol/L) & $1.30 \pm 0.36$ & $1.35 \pm 0.44$ & $1.31 \pm 0.39$ \\
\hline LDL (mmol/L) & $2.63 \pm 1.14$ & $2.35 \pm 0.98$ & $2.54 \pm 1.09$ \\
\hline TG (mmo/L) & $1.71 \pm 1.01$ & $1.88 \pm 1.06$ & $1.77 \pm 1.03$ \\
\hline
\end{tabular}

BMI - Body Mass Index, HbAlc - glycated haemoglobin, FBG-Fasting blood glucose, TC - Total Cholesterol, HDL - high-density lipoproteins, LDL - Low Density Lipoproteins, TG - Triglycerides, CRT - Creatinine, GFR - glomerular filtration rate. $\mathrm{n}$ - Total number of samples/patients, P-value $>0.05 .{ }^{*} \mathrm{HbAlc}$ and RBG were

only measured for patients diagnosed with DM thus $\mathrm{n}$ vary. Values are presented as means \pm standard deviation

Table 2 and 3 indicates the socio-demographic and modifiable risk factors of non-diabetic and diabetic groups as well as among non-hypertensive and hypertensive groups. It is important to note that approximately $40 \%$ of the study cohort was co-morbid. In both NCDs, the proportion of females is higher than males, however, sex was only shown to be significantly associated amongst diabetic patients ( $\mathrm{p}$-value $=0.043$ ) Amongst diabetic patients, smoking status; salt intake, TG and HDL were all significantly associated with disease incidence with p-values of $0.015 ; 0.004,0.012$ and 0.003 respectively (Table 2 ). All other factors, i.e. age, 
educational level, physical activity, alcohol consumption, TC and LDL were not significantly associated with DM (Table 2). BMI was the only modifiable risk factor that showed significant association amongst hyperten- sive patients with a p-value of $<0.0001$ (Table 3). Factors not significantly associated with HTN were: sex, age, educational level, smoking status, physical activity, salt intake, and alcohol consumption, TC, TG, LDL and HDL (Table 3).

Table 2: Socio-demographics and Modifiable risk factors among diabetes $(\mathrm{n}=\mathbf{2 6 5})$.

\begin{tabular}{|c|c|c|c|c|c|c|}
\hline \multirow{3}{*}{ Variables } & \multirow{3}{*}{ Subgroups } & \multicolumn{4}{|c|}{ Group } & \multirow{3}{*}{$X^{2}, P$ Value } \\
\hline & & \multicolumn{2}{|c|}{ Non-diabetic } & \multicolumn{2}{|c|}{ Diabetic } & \\
\hline & & $n=148$ & $\%$ & $\mathrm{n}=117$ & $\%$ & \\
\hline \multirow{2}{*}{ Gender } & Male & 58 & 64.4 & 32 & 35.5 & \multirow[b]{2}{*}{$4.08,0.040$} \\
\hline & Female & 90 & 51.4 & 85 & 48.6 & \\
\hline \multirow{2}{*}{ Age } & Less than 50 years & 28 & 63.6 & 16 & 36.4 & \multirow[b]{2}{*}{$1.30,0.260$} \\
\hline & More than 50 years & 120 & 54.3 & 101 & 45.7 & \\
\hline \multirow{4}{*}{$\begin{array}{l}\text { Educational } \\
\text { level }\end{array}$} & No formal education & 12 & 60.0 & 8 & 40.0 & \multirow{4}{*}{$4.78,0.190$} \\
\hline & Primary education & 34 & 54.0 & 29 & 46.0 & \\
\hline & Secondary education & 97 & 58.1 & 70 & 41.9 & \\
\hline & Higher education & 4 & 28.6 & 10 & 71.4 & \\
\hline \multirow{3}{*}{$\begin{array}{l}\text { Smoking } \\
\text { status }\end{array}$} & Never smoked & 99 & 52.1 & 91 & 47.9 & \multirow{3}{*}{$8.45,0.015$} \\
\hline & Quit smoking & 29 & 56.9 & 22 & 43.1 & \\
\hline & Current smoker & 20 & 83.3 & 4 & 16.7 & \\
\hline \multirow{3}{*}{$\begin{array}{l}\text { Physical } \\
\text { activity }\end{array}$} & $\begin{array}{l}\text { More than } 3 \text { times / } \\
\text { week }\end{array}$ & 8 & 50.0 & 8 & 50.0 & \multirow{3}{*}{$0.25,0.880$} \\
\hline & 1-2 times/ week & 119 & 54.6 & 92 & 43.4 & \\
\hline & No physical activities & 21 & 55.3 & 17 & 44.7 & \\
\hline \multirow{3}{*}{ Salt intake } & No salt intake & 11 & 37.9 & 18 & 62.1 & \multirow{3}{*}{$11.15,0.004$} \\
\hline & Normal salt intake & 103 & 53.9 & 88 & 46.1 & \\
\hline & Increased salt intake & 34 & 75.6 & 11 & 24.4 & \\
\hline \multirow{3}{*}{$\begin{array}{c}\text { Alcohol } \\
\text { consumption }\end{array}$} & Never drank & 78 & 54.9 & 64 & 45.1 & \multirow{3}{*}{$3.63,0.160$} \\
\hline & Quit drinking & 40 & 50.6 & 39 & 49.4 & \\
\hline & Occasional drinker & 30 & 68.2 & 14 & 31.8 & \\
\hline \multirow{4}{*}{$\begin{array}{c}\text { BMI } \\
\left(\mathrm{Kg} / \mathrm{m}^{2}\right)\end{array}$} & $<18.5$ & 4 & 57.1 & 3 & 42.9 & \multirow{4}{*}{$0.28,0.960$} \\
\hline & $18.5-24.9$ & 21 & 55.3 & 17 & 44.7 & \\
\hline & $25.0-29.9$ & 37 & 53.6 & 32 & 46.4 & \\
\hline & $\geq 30$ & 86 & 57.3 & 64 & 42.7 & \\
\hline \multirow{2}{*}{$\begin{array}{c}\mathrm{TC} \\
(\mathrm{mmol} / \mathrm{L})\end{array}$} & Increased & 52 & 46.8 & 59 & 53.2 & \multirow[b]{2}{*}{$0.15,0.700$} \\
\hline & Normal & 96 & 56.8 & 58 & 43.2 & \\
\hline \multirow{2}{*}{$\begin{array}{c}\mathrm{TG} \\
(\mathrm{mmol} / \mathrm{L}) \\
\end{array}$} & Increased & 52 & 46.3 & 59 & 53.7 & \multirow[b]{2}{*}{$6.28,0.012$} \\
\hline & Normal & 95 & 62.3 & 58 & 37.7 & \\
\hline \multirow{2}{*}{$\begin{array}{c}\mathrm{HDL} \\
(\mathrm{mmol} / \mathrm{L})\end{array}$} & Decreased & 74 & 67.3 & 36 & 32.7 & \multirow[b]{2}{*}{$8.81,0.003$} \\
\hline & Normal & 69 & 48.6 & 73 & 51.4 & \\
\hline LDL & Increased & 50 & 56.8 & 38 & 43.2 & \\
\hline$(\mathrm{mmol} / \mathrm{L})$ & Normal & 98 & 55.4 & 79 & 44.6 & $0.05,0.820$ \\
\hline
\end{tabular}

$\mathrm{TC}=$ Total Cholesterol, $\mathrm{TG}=$ Triglyceride, $\mathrm{HDL}=$ High density lipoprotein, $\mathrm{LDL}=$ Low density lipoprotein, mmol= mill mole, L= litre. Location: Mdantsane, Eastern Cape (July 2018) 
Table 3: Factors affecting the Modifiable risk factors of hypertension in study subjects $(\mathrm{n}=265)$.

\begin{tabular}{|c|c|c|c|c|c|c|}
\hline \multirow{3}{*}{ Variables } & \multirow{3}{*}{ Subgroups } & \multicolumn{4}{|c|}{ Group } & \multirow{3}{*}{$X^{2}, P$ value } \\
\hline & & \multicolumn{2}{|c|}{ Non-hypertensive } & \multicolumn{2}{|c|}{ Hypertensive } & \\
\hline & & $n=13$ & $\%$ & $n=252$ & $\%$ & \\
\hline \multirow[t]{2}{*}{ Gender } & Male & 6 & 6.6 & 84 & 93.3 & \multirow[t]{2}{*}{$0.91,0.34$} \\
\hline & Female & 7 & 4 & 168 & 96 & \\
\hline \multirow[t]{2}{*}{ Age } & Less than 50 years & 4 & 9.1 & 40 & 90.9 & \multirow[t]{2}{*}{$1.98,0.16$} \\
\hline & More than 50 years & 9 & 4.1 & 212 & 95.9 & \\
\hline \multirow{4}{*}{$\begin{array}{l}\text { Educational } \\
\text { level }\end{array}$} & Uneducated & 0 & 0 & 20 & 100 & \multirow{4}{*}{$1.46,0.69$} \\
\hline & Primary & 4 & 6.3 & 59 & 93.7 & \\
\hline & Secondary & 8 & 4.8 & 159 & 95.2 & \\
\hline & High education & 1 & 7.1 & 13 & 92.9 & \\
\hline \multirow{3}{*}{$\begin{array}{l}\text { Smoking } \\
\text { status }\end{array}$} & Never smokers & 8 & 4.2 & 182 & 95.8 & \multirow{3}{*}{$1.17,0.56$} \\
\hline & Quit smokers & 4 & 7.8 & 47 & 92.2 & \\
\hline & Current smokers & 1 & 4.2 & 23 & 95.8 & \\
\hline \multirow[t]{3}{*}{$\begin{array}{l}\text { Physical } \\
\text { activity }\end{array}$} & $\begin{array}{l}\text { More than } 3 \text { times / } \\
\text { week }\end{array}$ & 0 & 11.1 & 16 & 88.9 & \multirow{3}{*}{$0.88,0.64$} \\
\hline & 1-2 times/ week & 11 & 4.3 & 200 & 95.7 & \\
\hline & No physical activities & 2 & 5.3 & 36 & 94.7 & \\
\hline \multirow[t]{3}{*}{ Salt intake } & No salt intake & 0 & 0 & 29 & 100 & \multirow{3}{*}{$1.84,0.40$} \\
\hline & Normal salt intake & 10 & 5.2 & 181 & 94.8 & \\
\hline & Increased salt intake & 3 & 6.7 & 42 & 93.3 & \\
\hline \multirow{3}{*}{$\begin{array}{l}\text { Alcohol } \\
\text { consumption }\end{array}$} & Never drank & 4 & 2.8 & 138 & 97.2 & \multirow{3}{*}{$3.32,0.19$} \\
\hline & Quit drinking & 5 & 6.3 & 74 & 93.7 & \\
\hline & Occasional drinker & 4 & 9.1 & 40 & 90.9 & \\
\hline \multirow[t]{4}{*}{ BMI (Kg/m²) } & $<18.5$ & 2 & 28.6 & 5 & 71.4 & \multirow{4}{*}{$\begin{array}{c}21.34 \\
<0.0001\end{array}$} \\
\hline & $18.5-24.9$ & 6 & 15.8 & 32 & 84.2 & \\
\hline & $25.0-29.9$ & 1 & 1.4 & 68 & 98.6 & \\
\hline & $\geq 30$ & 4 & 2.7 & 146 & 97.3 & \\
\hline \multirow[t]{2}{*}{$\mathrm{TC}$ (mmol/L) } & Increased & 5 & 4.9 & 98 & 95.1 & \multirow[b]{2}{*}{$0.001,0.98$} \\
\hline & Normal & 8 & 4.9 & 154 & 95.1 & \\
\hline \multirow[t]{2}{*}{ TG (mmol/L) } & Increased & 4 & 3.5 & 107 & 96.5 & \multirow[b]{2}{*}{$0.69,0.41$} \\
\hline & Normal & 9 & 5.8 & 145 & 94.2 & \\
\hline \multirow[t]{2}{*}{ HDL (mmol/L) } & Decreased & 2 & 1.8 & 108 & 98.2 & \multirow[b]{2}{*}{$2.37,0.12$} \\
\hline & Normal & 8 & 5.6 & 134 & 94.4 & \\
\hline LDL (mmol/L) & Increased & 4 & 4.5 & 84 & 95.5 & \\
\hline & Normal & 9 & 5.1 & 168 & 94.9 & $0.04,0.85$ \\
\hline
\end{tabular}

Table 4 and 5 describe the univariate and multivariate analyses for diabetes and hypertension. The univariate results show a risk association between diabetes and smoking status, salt intake, TG and HDL (Table 4). The results gave an indication that smoking status has an impact on diabetes using never smoked as the reference, those who quit smoking had significantly lesser odds $(p$-value $=0.824)$ of diabetes in comparison to those who are currently smokers ( $\mathrm{p}$-value $=0.048)$ (Table 4). Furthermore, an increased salt intake, increased TG and a decreased HDL-C also demonstrated significant- ly higher odds ( $\mathrm{p}$-value $=0.01$ ) of diabetes. Gender was not significantly associated with diabetes ( $\mathrm{p}$-value $=0.210)$. In table 5 , an increased $\mathrm{BMI}$ in comparison to the underweight (as per WHO standards) has higher odds of hypertension ( $\mathrm{p}$-value $=0.012$ and $\mathrm{p}$-value $=0.006$ ). In addition to this, participants who have normal BMI have lower odds of hypertension ( $p$-value $=0.420$ ).

Multivariate logistic regression analysis showed that after adjusting for all significant factors, an increased salt intake and BMI were significantly associated with DM and HTN respectively (Table 4 and 5). 
Table 4: Univariate and Multivariate analysis for risk factors of Diabetes status

\begin{tabular}{|c|c|c|c|c|c|}
\hline Factors & $\begin{array}{c}\text { Diabetic } \\
\text { N }(\%)\end{array}$ & $\begin{array}{c}\text { Non- } \\
\text { Diabetic } \\
\text { N (\%) } \\
\end{array}$ & $\begin{array}{l}\text { Unadjusted } \\
\text { Odds ratio }\end{array}$ & $\begin{array}{c}\text { Adjusted } \\
\text { odds ratio }\end{array}$ & P-value \\
\hline \multicolumn{6}{|c|}{ Sex } \\
\hline Male & $32(35.6)$ & $58(64.4)$ & 1 & 1 & \\
\hline Female & $90(51.4)$ & $85(48.6)$ & $\begin{array}{c}1.71(1.01- \\
2.89)^{*}\end{array}$ & $\begin{array}{c}1.55(0.78- \\
3.1)\end{array}$ & 0.212 \\
\hline \multicolumn{6}{|c|}{ Salt intake } \\
\hline $\begin{array}{l}\text { No salt } \\
\text { intake }\end{array}$ & $18(62.1)$ & $11(37.9)$ & 1 & 1 & 0.009 \\
\hline $\begin{array}{l}\text { Normal salt } \\
\text { intake }\end{array}$ & $88(46.1)$ & $103(53.9)$ & $\begin{array}{c}0.52(0.23- \\
1.17)\end{array}$ & $\begin{array}{c}0.54(0.19- \\
1.08)\end{array}$ & 0.075 \\
\hline $\begin{array}{l}\text { increased salt } \\
\text { intake }\end{array}$ & $11(24.4)$ & $34(75.6)$ & $\begin{array}{c}0.2(0.07- \\
0.54)^{*}\end{array}$ & $\begin{array}{c}0.18(0.06- \\
0.55)^{*}\end{array}$ & $0.002 *$ \\
\hline \multicolumn{6}{|c|}{ Smoking status } \\
\hline Never smoke & $91(47.9)$ & $99(52.1)$ & 1 & 1 & \\
\hline Quit smoking & $22(43.1)$ & $29(56.9)$ & $\begin{array}{c}0.83(0.44- \\
1.54) \\
\end{array}$ & $\begin{array}{c}1.09(0.51- \\
2.36) \\
\end{array}$ & 0.824 \\
\hline $\begin{array}{l}\text { Current } \\
\text { smokers }\end{array}$ & $4(16.7)$ & $20(83.3)$ & $\begin{array}{c}0.22(0.07- \\
0.66)^{*}\end{array}$ & $\begin{array}{c}0.26(0.07- \\
0.98)\end{array}$ & $0.048^{*}$ \\
\hline \multicolumn{6}{|c|}{ TG- Cholesterol } \\
\hline Normal level & $58(37.7)$ & $96(62.3)$ & 1 & 1 & \\
\hline $\begin{array}{l}\text { Increased } \\
\text { level }\end{array}$ & $59(53.2)$ & $52(46.8)$ & $\begin{array}{c}1.88(1.15- \\
3.08)^{*} \\
\end{array}$ & $\begin{array}{c}2.19(1.3- \\
3.8) \\
\end{array}$ & $0.006^{*}$ \\
\hline \multicolumn{6}{|c|}{ HDL-Cholesterol } \\
\hline Normal level & $73(51.4)$ & $69(48.6)$ & 1 & 1 & \\
\hline $\begin{array}{l}\text { Decreased } \\
\text { level }\end{array}$ & $36(32.7)$ & $74(67.3)$ & $\begin{array}{c}1.06(0.27- \\
0.77)^{*}\end{array}$ & $\begin{array}{c}0.38(0.22- \\
0.67) \\
\end{array}$ & $0.001 *$ \\
\hline
\end{tabular}

*P-value $<0.05$.

Location: Mdantsane, Eastern Cape (July 2018) 
Table 5: Univariate and Multivariate analysis for risk factors of hypertension

\begin{tabular}{|c|c|c|c|c|c|}
\hline Factors & $\begin{array}{c}\text { Hypertensive } \\
\text { N (\%) }\end{array}$ & $\begin{array}{c}\text { Non- } \\
\text { hypertensive } \\
\text { N (\%) }\end{array}$ & $\begin{array}{l}\text { Unadjusted } \\
\text { Odds ratio }\end{array}$ & $\begin{array}{l}\text { Adjusted } \\
\text { odds } \\
\text { ratio } \\
\end{array}$ & P-value \\
\hline \multicolumn{6}{|c|}{ BMI } \\
\hline$<18.5$ & $5(71.4)$ & $2(28.6)$ & 1 & 1 & \\
\hline $18.5-24.9$ & $32(84.2)$ & $6(15.8)$ & $\begin{array}{c}0.47(0.07- \\
3.0)\end{array}$ & $\begin{array}{c}0.35 \\
(0.048- \\
2.56) \\
\end{array}$ & 0.304 \\
\hline $25.0-29.9$ & $68(98.6)$ & $1(1.4)$ & $\begin{array}{l}0.04(0.003- \\
0.48)^{*}\end{array}$ & $\begin{array}{c}0.03 \\
(0.002- \\
0.44)^{*} \\
\end{array}$ & $0.010^{*}$ \\
\hline$\geq 30$ & $146(97.3)$ & $4(2.7)$ & $\begin{array}{c}0.07(0.01- \\
0.47)^{*}\end{array}$ & $\begin{array}{c}0.06 \\
(0.007- \\
0.447)^{*}\end{array}$ & $0.006^{*}$ \\
\hline \multicolumn{6}{|c|}{ Smoking status } \\
\hline $\begin{array}{l}\text { Never } \\
\text { smoke }\end{array}$ & $182(95.8)$ & $8(4.2)$ & 1 & 1 & \\
\hline $\begin{array}{l}\text { Quit } \\
\text { smoking }\end{array}$ & $47(92.2)$ & $4(7.8)$ & $\begin{array}{c}1.93(0.60- \\
6.7)\end{array}$ & $\begin{array}{c}0.764 \\
(0.16- \\
3.61) \\
\end{array}$ & 0.700 \\
\hline $\begin{array}{l}\text { Current } \\
\text { smokers }\end{array}$ & $23(95.8)$ & $1(4.2)$ & $\begin{array}{c}0.99(0.012- \\
8.3)\end{array}$ & $\begin{array}{c}0.34 \\
(0.04- \\
3.9) \\
\end{array}$ & 0.400 \\
\hline \multicolumn{6}{|c|}{ Alcohol consumption } \\
\hline $\begin{array}{l}\text { Never } \\
\text { drank }\end{array}$ & $138(97.2)$ & $4(2.8)$ & 1 & 1 & \\
\hline $\begin{array}{l}\text { Quit } \\
\text { drinking }\end{array}$ & $74(93.7)$ & $5(6.3)$ & $\begin{array}{c}2.33(0.61- \\
8.95) \\
\end{array}$ & $\begin{array}{c}3.7(0.67- \\
20.6) \\
\end{array}$ & 0.14 \\
\hline $\begin{array}{l}\text { Occasional } \\
\text { drinker }\end{array}$ & $40(90.9)$ & $4(9.1)$ & $\begin{array}{c}3.45(0.83- \\
14.4)\end{array}$ & $\begin{array}{c}2.01 \\
(0.42- \\
9.67) \\
\end{array}$ & 0.38 \\
\hline
\end{tabular}

\section{Discussion}

South Africa has been reported to have the highest incidence of DM in the African continent ${ }^{14}$. Amongst the modifiable risk factors, significant association was shown with tobacco intake, increased salt intake, TG and HDL. Tobacco smoking is well established as a risk factor for multiple diseases and has been associated with DM in multiple cohort studies ${ }^{32-34}$. The present study showed that smoking was associated with the probability of developing DM. This finding is consistent with previous studies conducted in Korea ${ }^{35 ; 36}$. Current smokers and ex-smokers display a greater probability of developing DM than non-smokers, however in this study, the increased risk of ex-smokers were not statistically significant. Previous studies conducted by Jee et al. ${ }^{35}$ and Hur et al. ${ }^{37}$ also reported the increased risk of ex-smokers as insignificant.

The WHO (2016) recommends that patients with DM should reduce their dietary salt intake ${ }^{2}$. The precise relationship between dietary salt intake and DM is not well defined, however, excessive salt intake is well associated with hypertension and CVDs. In the present study, an increased salt intake was significantly associated with the higher incidence of DM. Previous studies also demonstrated an association between high dietary salt intake and $\mathrm{DM}^{38-40}$. Increased TG levels have been associated with an increased risk of $\mathrm{DM}^{41-44}$ and in this study cohort, similar results were observed.

In addition, this study also found that the odds of having DM were increased with a decrease in HDL. Similar findings have been reported in African ${ }^{45-48}$, European ${ }^{49}$ and United States communities ${ }^{50 ; 51}$. Lower levels of HDL concentrations have been associated with many diseases such as CVDs ${ }^{52-55}$, nephropathy ${ }^{56}$ and coronary heart disease ${ }^{57 ; 58}$. Although, levels of TC and LDL in diabetic individuals are reportedly comparable with that found in non-diabetics, low levels of HDL and elevated TG have been reported in T2DM patients as the probable cause of $\mathrm{CVD}^{52-54}$. It has also been observed that HDL alone might not be a good indicator of increase DM risk since most of the subjects had lower total cholesterol. Moreover, lower levels of HDL in the present study might be because of the lower cholesterol.

A high BMI is a risk factor that is often associated with 
$\mathrm{DM}^{59}$, however, in this study; it was significantly associated with HTN, since DM and HTN co-exist in approximately $40 \%$ of the study cohort, this could be an explanation for this observation. Furthermore, many studies suggest that a high BMI contributes to hypertension ${ }^{60-64}$. It is well established that smoking increases the risk of hypertension; however, the significance of this association may differ between populations $6^{5}$. In this study, no significant association was observed between hypertension and smoking status. These findings are contrary to other studies ${ }^{66-69}$.

The following limitations need to be considered; the cross-sectional design and recruitment of participants from one study centre might limit the generalisation of the findings. In addition, the scope of this study needs more samples drawn from broader population across the country to conclude on an association that exists between DM and HTN with the discussed variables.

\section{Conclusion}

DM was associated with smoking and salt intake; whilst hypertension was associated with increasing BMI. Development of best practices for affordable and effective programs in screening, prevention, detection and treatment of DM and HTN is essential. In order to reduce the burden of NCDs, comprehensive intervention strategies should be implemented across the country. Future studies with larger sample size should be done to identify or generate local modifiable risk factors for the development of DM and HTN.

\section{Acknowledgments}

The authors would also like to thank the study participants, Cecilia Makiwane Hospital and the Department of Health Eastern Cape. The work reported herein was made possible through funding by the South African Medical Research Council through its Division of Research Capacity Development under funding received from the South African National Treasury. The content hereof is the sole responsibility of the authors and do not necessarily represent the official views of the SAM$\mathrm{RC}$ or the funders. In addition, partial funding from the National Research Foundation of South African was used for this study.

\section{Ethical clearance}

Ethical approval for this study was approved by the ethics committees of the University of the Western Cape
(UWC) and the Eastern Cape Department of Health (Ethics Reference Number: BM/16/5/19).

\section{Declaration of authorship}

MB, LX, BP, OVA, RJ conceived and designed the study; LX acquired and prepared the data; LX and MMM analysed and interpreted the data; LX and ZAO drafted the manuscript; LX, ZAO and MMM revised the manuscript for important intellectual content; all authors gave approval of the version to be submitted and agree to be accountable for all aspects of the work.

\section{Competing interests}

All authors have completed the Unified Competing Interest form at www.icmje.org/coi_disclosure.pdf (available on request from the corresponding author) and declare: no support from any organization for the submitted work; no financial relationships with any organizations that might have an interest in the submitted work in the previous 3 years; no other relationships or activities that could appear to have influenced the submitted work.

\section{References}

1. van de Vijver S, Akinyi H, Oti S, Olajide A, Agyemang C, Aboderin I, et al. Status report on hypertension in Africa-Consultative review for the 6th Session of the African Union Conference of Ministers of Health on NCD's. Pan African Medical Journal. 2014; 16(1).

2. World Health Organization Global report on diabetes: World Health Organization. 2016.

3. Alberts M, Urdal P, Steyn K, Stensvold I, Tverdal A, Nel JH, Steyn NP. Prevalence of cardiovascular diseases and associated risk factors in a rural black population of South Africa. European Journal of Cardiovascular Prevention \& Rehabilitation. 2005 Aug; 12(4):347-54.

4. Puoane T, Tsolekile L, Caldbick S, Igumbor E, Meghnath K, Sanders D. Chronic non-communicable diseases in South Africa: Progress and challenges. South African Health Review. 2012; 13:115-126.

5. Maimela E, Alberts M, Modjadji SE, Choma SS, Dikotope SA, Ntuli TS, Van Geertruyden J. The prevalence and determinants of chronic non-communicable disease risk factors amongst adults in the Dikgale health demographic and surveillance system (HDSS) site, Limpopo Province of South Africa. PloS one. 2016; 11(2): e0147926.

6. World Health Organization Global status report on noncommunicable diseases. Geneva: 2010. World Health Organization: 2015. 
7. Kearney PM, Whelton M, Reynolds K, Muntner P, Whelton PK, He J. Global burden of hypertension: analysis of worldwide data. The Lancet. 2005 Jan 15; 365(9455):217-23.

8. Opie LH, Seedat YK. Hypertension in sub-Saharan African populations. Circulation. 2005 Dec 6; 112(23):3562-8.

9. Centers for Disease Control and Prevention (CDC. Vital signs: awareness and treatment of uncontrolled hypertension among adults--United States, 2003-2010. MMWR. Morbidity and Mmortality Weekly Report. 2012 Sep 7; 61:703.

10. World Health Organization. World health statistics 2012 report. World Health Organization. Geneva 2012 11. American Diabetes Association. Standards of medical care in diabetes-2014. Diabetes Care. 2014 Jan 1; 37 Suppl 1:S14-80.

12. Pradeep T, Haranath C. A Review on Diabetes Mellitus Type II. International Journal of Pharma Research \& Review. 2014 Sep; 3(9):23-9.

13. Dokunmu TM, Yakubu OF, Adebayo AH, Olasehinde G.I, Chinedu SN. Cardiovascular Risk Factors in a Suburban Community in Nigeria. International Journal of Hypertension. 2018; 2018.

14. Atlas ID. 2016. International Diabetes Federation $7^{\text {th }}$ Edition, 2015,

15. Todd JN, Florez JC. An update on the pharmacogenomics of metformin: progress, problems and potential. Pharmacogenomics. 2014 Mar; 15(4):529-39.

16. Williams B. Insulin resistance: the shape of things to come. The Lancet. 1994 Aug 20; 344(8921):521-4.

17. World Health Organization. Global action plan for the prevention and control of noncommunicable diseases 2013-2020. 2013. Geneva: World Health Organization. 2015.

18. World Health Organization \& International Society of Hypertension Writing Group. 2003 World Health Organization (WHO)/International Society of Hypertension (ISH) statement on management of hypertension. Journal of Hypertension. 2003; 21(11): 1983-1992.

19. Diabetes Prevention Program Research Group. Reduction in the incidence of type 2 diabetes with lifestyle intervention or metformin. New England Journal of Medicine. 2002 Feb 7; 346(6):393-403.

20. Griffin SJ, Borch-Johnsen K, Davies MJ, Khunti K, Rutten GE, Sandbæk A, Sharp SJ, Simmons RK, Van den Donk M, Wareham NJ, Lauritzen T. Effect of early intensive multifactorial therapy on 5 -year cardiovascular outcomes in individuals with type 2 diabetes detected by screening (ADDITION-Europe): a cluster-randomised trial. The Lancet. 2011 Jul 9; 378(9786):156-67.
21. Herman WH, Ye W, Griffin SJ, Simmons RK, Davies MJ, Khunti K, Rutten GE, Sandbaek A, Lauritzen T, Borch-Johnsen K, Brown MB. Early detection and treatment of type 2 diabetes reduce cardiovascular morbidity and mortality: a simulation of the results of the Anglo-Danish-Dutch Study of Intensive Treatment in People With Screen-Detected Diabetes in Primary Care (ADDITION-Europe). Diabetes Care. 2015 May 15:dc142459.

22. Forouzanfar MH, Afshin A, Alexander LT, Anderson HR, Bhutta ZA, Biryukov S, Brauer M, Burnett R, Cercy K, Charlson FJ, Cohen AJ. Global, regional, and national comparative risk assessment of 79 behavioural, environmental and occupational, and metabolic risks or clusters of risks, 1990-2015: a systematic analysis for the Global Burden of Disease Study 2015. The Lancet. 2016 Oct 8; 388(10053):1659-1724.

23. Adeniyi OV, Yogeswaran P, Longo-Mbenza B, Ter Goon D. Uncontrolled hypertension and its determinants in patients with concomitant type 2 diabetes mellitus (T2DM) in rural South Africa. PLoS One. 2016 Mar 1; 11(3):e0150033.

24. World Health Organization \& World Health Organization. Burden: mortality, morbidity and risk factors. Global Status Report on Non-communicable Disease. 2010; 2011.

25. Mayosi BM, Flisher AJ, Lalloo UG, Sitas F, Tollman SM, Bradshaw D. The burden of non-communicable diseases in South Africa. The Lancet. 2009 Sep 12; 374(9693):934-47.

26. Ntuli ST, Alberts M, Choma S, Dikotope S, Maimela E. Prevalence and associated risk factors of hypertension amongst adults in a rural community of Limpopo Province, South Africa. African Journal of Primary Health Care and Family Medicine. 2015; 7(1): 1-5.

27. Stats S. Statistics South Africa. Formal census. 2011 28. The Heart and Stroke Foundation, South Africa. The Heart and Stroke Foundation, South Africa 2017. Updated November 2018; cited 19 June 2018. Available from: http://www.heartfoundation.co.za/cholesterol/. 29. Amod A. The 2012 SEMDSA guideline for the management of type 2 diabetes. Journal of Endocrinology, Metabolism and Diabetes in South Africa. 2012 Jan 1; 17(1):61-2.

30. World Health Organization. World health statistics 2015. World Health Organization. 2015

31. Atlas ID. International Diabetes Federation 8th Edition, 2017. 2017

32. US Department of Health and Human Services. The health consequences of smoking: a report of the Surgeon General, 2004. 
33. Willi C, Bodenmann P, Ghali WA, Faris PD, Cornuz J. Active smoking and the risk of type 2 diabetes: a systematic review and meta-analysis. JAMA. 2007; 298(22): 2654-2664.

34. Cassano PA, Rosner B, Vokonas PS, Weiss ST. Obesity and Body Fat Distribution in Relation to the Incidence of Non-lnsulin-dependent Diabetes Mellitus: A Prospective Cohort Study of Men in the Normative Aging Study. American Journal of Epidemiology. 1992; 136(12): 1474-1486.

35. Jee SH, Foong AW, Hur NW, Samet JM. Smoking and risk for diabetes incidence and mortality in Korean men and women. Diabetes Car. 2010; 33(12): 2567-2572. 36. Kim HC, Oh SM. Noncommunicable diseases: current status of major modifiable risk factors in Korea. Journal of preventive medicine and public health. Yebang Uibakhoe chi. 2013; 46 (4): 165-172.

37. Hur NW, Kim HC, Mo Nam C, Ha Jee S, Lee HC, Suh I. Smoking cessation and risk of type 2 diabetes mellitus: Korea Medical Insurance Corporation Study. European Journal of Cardiovascular Prevention \& Rebabilitation. 2007; 14(2): 244-249.

38. Hu G, Jousilahti P, Peltonen M, Lindström J, Tuomilehto J. Urinary sodium and potassium excretion and the risk of type 2 diabetes: a prospective study in Finland. Diabetologia. 2005; 48(8): 1477-1483.

39. Ekinci EI, Cheong K, Dobson M, Premaratne E, Finch S, Macisaac RJ, Jerums G. High sodium and low potassium intake in patients with type 2 diabetes. Diabetic Medicine. 2010; 27 (12): 1401-1408.

40. Ekinci EI, Clarke S, Thomas M.C, Moran JL, Cheong K, MacIsaac RJ, et al.. Dietary salt intake and mortality in patients with type 2 diabetes. Diabetes Care. 2011; 34(3): 703-709.

41. Circulating cholesterol level and risk of death from cancer in men aged 40 to 69 years. Experience of an international collaborative group. JAMA. 1982; 248(21): 2853-2859.

42. Nomura AM, Stemmermann GN, Chyou P. Prospective study of serum cholesterol levels and large-bowel cancer. JNCI: Journal of the National Cancer Institute. 1991; 83(19): 1403-1407.

43. Sorlie PD, Feinleib M. The serum cholesterol-cancer relationship: an analysis of time trends in the Framingham Study. Journal of the National Cancer Institute.1982; 69(5): 989-996.

44. Williams RR, Sorlie PD, Feinleib M, McNamara PM, Kannel WB, Dawber TR. Cancer incidence by levels of cholesterol. JAMA.1981; 245(3): 247-252.

45. Motala AA, Esterhuizen T, Pirie FJ, Omar MA. The prevalence of metabolic syndrome and determination of the optimal waist circumference cutoff points in a rural South African community. Diabetes Care. 2011 Feb 14:DC_101921.

46. Tagoe DN, Amo-Kodieh P. Type 2 diabetes mellitus influences lipid profile of diabetic patients. Annals of Biological Research. 2013; 4(6):88-92.

47. Sumner AE, Zhou J, Doumatey A, Imoisili OE, Amoah A, Acheampong J, Oli J, Johnson T, Adebamowo C, Rotimi CN. Low HDL-cholesterol with normal triglyceride levels is the most common lipid pattern in West Africans and African Americans with metabolic syndrome: implications for cardiovascular disease prevention. CVD Prevention and Control. 2010 Sep 1; 5(3):75-80.

48. Fagot-Campagna AN, Knowler WC, Narayan KM, Hanson RL, Saaddine J, Howard BV. HDL cholesterol subfractions and risk of developing type 2 diabetes among Pima Indians. Diabetes Care 1999 Feb 1; 22(2):271-4.

49. Njølstad I, Amesen E, Lund-Larsen PG. Sex differences in risk factors for clinical diabetes mellitus in a general population: a 12-year follow-up of the Finnmark Study. American Journal of Epidemiology. 1998 Jan 1; 147(1):49-58.

50. Haffner SM, Stern MP, Hazuda HP, Mitchell BD, Patterson JK. Cardiovascular risk factors in confirmed prediabetic individuals: does the clock for coronary heart disease start ticking before the onset of clinical diabetes?. JAMA. 1990 Jun 6; 263(21):2893-8.

51. Montonen J, Drogan D, Joost HG, Boeing H, Fritsche A, Schleicher E, Schulze MB, Pischon T. Estimation of the contribution of biomarkers of different metabolic pathways to risk of type 2 diabetes. European Journal of Epidemiology. 2011 Jan 1; 26(1):29-38.

52. Gordon T, Castelli WP, Hjortland MC, Kannel WB, Dawber TR. High density lipoprotein as a protective factor against coronary heart disease: the Framingham Study. The American Journal of Medicine. 1977 May 1; 62(5):707-14.

53. Gordon DJ, Probstfield JL, Garrison RJ, Neaton JD, Castelli WP, Knoke JD, Jacobs Jr DR, Bangdiwala S, Tyroler HA. High-density lipoprotein cholesterol and cardiovascular disease. Four prospective American studies. Circulation. 1989 Jan; 79(1):8-15.

54. Sharrett AR, Ballantyne CM, Coady SA, Heiss G, Sorlie PD, Catellier D, Patsch W. Coronary heart disease prediction from lipoprotein cholesterol levels, triglycerides, lipoprotein (a), apolipoproteins $\mathrm{AI}$ and $\mathrm{B}$, and HDL density subfractions: The Atherosclerosis Risk 
in Communities (ARIC) Study. Circulation. 2001 Sep 4; 104(10):1108-13.

55. Karadag F, Ozcan H, Karul AB, Yilmaz M, Cildag O. Sex hormone alterations and systemic inflammation in chronic obstructive pulmonary disease. International Journal of Clinical Practice. 2009 Feb; 63(2):275-81.

56. Morton J, Zoungas S, Li Q, Patel AA, Chalmers J, Woodward M, Celermajer DS, Beulens JW, Stolk RP, Glasziou P, Ng MK. Low HDL cholesterol and the risk of diabetic nephropathy and retinopathy: results of the ADVANCE study. Diabetes Care. 2012 Aug 10:DC_120306.

57. Kucharska-Newton AM, Rosamond WD, Schroeder JC, McNeill AM, Coresh J, Folsom AR. HDL-cholesterol and the incidence of lung cancer in the Atherosclerosis Risk in Communities (ARIC) study. Lung Cancer. 2008 Sep 1; 61(3):292-300.

58. Filippatos TD, Elisaf MS. High density lipoprotein and cardiovascular diseases. World Journal of Cardiology. 2013 Jul 26; 5(7):210.

59. Al-Nsour M, Zindah M, Belbeisi A, Hadaddin R, Brown DW, Walke H. 2012, Prevalence of selected chronic, noncommunicable disease risk factors in Jordan: results of the 2007 Jordan Behavioral Risk Factor Surveillance Survey. Preventing Chronic Disease. 2018; 9: E25.

60. Vasan RS, Beiser A, Seshadri S, Larson MG, Kannel WB, D'agostino RB, Levy D. Residual lifetime risk for developing hypertension in middle-aged women and men: The Framingham Heart Study. JAMA.2002; 287(8): 1003-1010.

61. Redon J, Cea-Calvo L, Moreno B, Monereo S, Gil-Guillen V, Lozano JV, et al. Independent impact of obesity and fat distribution in hypertension prevalence and control in the elderly. Journal of Hypertension. 2008; 26(9): 1757-1764.
62. Rudatsikira E, Muula AS, Mulenga D, Siziya S. Prevalence and correlates of obesity among Lusaka residents, Zambia: a population-based survey. International Archives of Medicine. 2012; 5(1): 14.

63. Mulenga D, Siziya S, Rudatsikira E, Mukonka V, Babaniyi $\mathrm{O}$, Songolo $\mathrm{P}$, et al .District specific correlates for hypertension in Kaoma and Kasama rural districts of Zambia. Rural Remote Health.2013; 13(3): 2345.

64. Reddy S, Prabhu G. Prevalence and risk factors of hypertension in adults in an Urban Slum, Tirupati, AP. Indian Journal of community medicine. 2005; 30(3): 84.

65. Dhungana RR, Pandey AR, Bista B, Joshi S, Devkota $S$. Prevalence and associated factors of hypertension: a community-based cross-sectional study in municipalities of Kathmandu, Nepal. International Journal of Hypertension, .2016: 2016.

66. Pandey A, Patni N, Sarangi S, Singh M, Sharma K, Vellimana A.K, et al. Association of exclusive smokeless tobacco consumption with hypertension in an adult male rural population of India. Tobacco induced diseases. 2009; 5(1):15.

67. Shanthirani C, Pradeepa R, Deepa R, Premalatha G, Saroja R, Mohan V. Prevalence and risk factors of hypertension in a selected South Indian population--the Chennai Urban Population Study. The Journal of the Association of Physicians of India. 2003; 51: 20-27.

68. SIngh RB, Fedacko J, Pella D, Macejova Z, Ghosh S, De AK, Begom R, et al. 2011, Prevalence and risk factors for prehypertension and hypertension in five Indian cities. Acta Cardiologica.2011; 66, no. 1, pp. 29-37. 69. Mohan V, Deepa M, Farooq S, Datta M, Deepa R. Prevalence, awareness and control of hypertension in Chennai-the Chennai urban rural epidemiology study (CURES-52). Journal of Association of Physicians of India. 2007; 55: 326-332. 